\title{
Interference Suppression with Antenna Arrays in OFDM Systems under Transceiver I/Q Imbalance
}

\author{
Aki Hakkarainen*, Janis Werner*, Kapil Dandekar ${ }^{\dagger}$ and Mikko Valkama* \\ ${ }^{*}$ Department of Electronics and Communications Engineering, Tampere University of Technology, Tampere, Finland \\ Emails: \{aki.hakkarainen, janis.werner, mikko.e.valkama\} @tut.fi \\ ${ }^{\dagger}$ Department of Electrical and Computer Engineering, Drexel University, Philadelphia, PA 19104, USA \\ Email: dandekar@coe.drexel.edu
}

\begin{abstract}
In this paper, we address the effects of radio frequency (RF) transceiver in-phase/quadrature-phase (I/Q) imbalance in transmission systems which are utilizing orthogonal frequency-division multiplexing (OFDM) waveforms. Special emphasis is on the analysis of external interference sources and their mitigation with receiver antenna array processing, assuming independent fading for the antenna elements. In addition, I/Q imbalance is assumed to be arbitrarily frequency selective and independent in different transceiver branches. We show that I/Q imbalance is especially harmful in the presence of strong interferers when conventional per-subcarrier processing is implemented on the receiver side. Based on these results, we propose a joint subcarrier processing where each of the subcarrier signals is combined with the signal at the image carrier. Such processing is shown to be very efficient in $I / Q$ imbalance mitigation as well as in the total interference suppression.
\end{abstract}

Index Terms-antenna arrays, in-phase/quadrature-phase (I/Q) imbalance, orthogonal frequency-division multiplexing (OFDM), uncorrelated fading

\section{INTRODUCTION}

Interference suppression is a very important topic in modern radio systems. E.g. in cognitive radio systems, primary users (PU) have to be protected from the interference caused by the secondary users (SU) for preventing system failures in the primary network [1]. In addition, SUs need to suppress strong interference coming from the PUs, in order to operate reliably within the same area. Such interference protection/suppression capabilities can be obtained e.g. with antenna arrays. With antenna array processing [2], a signal can be transmitted/received by several antennas and thus the data link can be steered towards the desired direction while nulling the influence to/from the non-desired directions. Unfortunately, imperfections in radio frequency (RF) electronics can be very harmful for these functionalities, see e.g. [3], [4].

This work is supported by the Finnish Funding Agency for Technology and Innovation (Tekes) under the project "Reconfigurable Antenna-based Enhancement of Dynamic Spectrum Access Algorithms", the Industrial Research Fund of Tampere University of Technology (Tuula and Yrjö Neuvo Fund), the Academy of Finland under the project 251138 "Digitally-Enhanced RF for Cognitive Radio Devices", the Doctoral Programme of the President of Tampere University of Technology, and the Foundation of Nokia Corporation.

The work is also supported by National Science Foundation (NSF) under award number 1147838 .
One of these RF imperfections is in-phase quadrature-phase (I/Q) imbalance [5] which is created in direct-conversion transceivers (DCT) [6]. It is distorting the signal properties and thus degrading the overall performance of the transmission system [5]. Despite of I/Q imbalance and other disadvantages, DCT is a very promising RF front-end implementation candidate for modern transmission systems due to the smaller size and cost than e.g. with the superheterodyne transceivers [7].

The importance of I/Q imbalance and its mitigation has risen when high data rate transmission systems, utilizing large symbol alphabets and orthogonal frequency-division multiplexing (OFDM) waveforms [8], have become more popular. A comprehensive analysis of $\mathrm{I} / \mathrm{Q}$ imbalance of a single data link in OFDM systems is given in [9], [10]. Analysis is extended to consider multiple transmitter (TX) antennas in [11] whereas [12], [13] include several antennas on both TX and receiver (RX) sides. In [14] the I/Q imbalance problem is studied together with power amplifier nonlinearities whereas [15] concentrates on the joint effects of I/Q imbalance and carrier frequency offset. However, the influence of external interference sources is not included in any of these analyses.

In this paper we analyze the influence of external interference in antenna array processing under transceiver I/Q imbalance. Analysis is done at the subcarrier level in order to keep the results applicable for general OFDM systems. We do not assume any specific dependency in the I/Q imbalance parameters between transceiver branches or subcarriers, and thus the I/Q imbalance parameters can be arbitrarily frequency selective. As proposed e.g. in [9], [13], we will use joint subcarrier processing as the key element in I/Q imbalance mitigation and interference suppression under I/Q imbalance. The proposed solution is based on a data-aided processing which is implemented in the RX side only and thus does not require any additional communication between RX and TX. It will be shown that the proposed solution provides effective interference suppression in spite of severe RF imperfections.

The paper is organized as follows. Section II presents TX and RX signal models under I/Q imbalance, and describes joint subcarrier processing. In Section III, an analytical evaluation is carried out with covariance matrices, output powers and signal to interference plus noise ratios (SINRs). Section IV describes how to optimize the receiver spatial array processing 
in the minimum mean square error (MMSE) sense. Numerical evaluations are given in Section V and finally, conclusions are drawn in Section VI.

Notation: Throughout this paper, vectors and matrices are written with bold characters. The superscripts $(\cdot)^{T},(\cdot)^{H},(\cdot)^{*}$ and $(\cdot)^{-1}$ represent transpose, Hermitian (conjugate) transpose, conjugate and matrix inverse, respectively. The tilde sign $(\cdot)$ is used to present an augmented quantity and the results obtained by the augmented processing. The statistical expectation is denoted with $\mathbb{E}[\cdot]$. A complex random variable $x$ is called circular if $\mathbb{E}\left[x^{2}\right]=0$.

\section{ESSENTIAL Signal MODELS}

\section{A. Signals with $T X$ and $R X I / Q$ Imbalances}

In OFDM transmission, a wide transmission band is divided into several orthogonal subcarriers which carry, in general, independent data streams [8]. These individual subcarrier signals can be considered as narrowband signals which have constant (flat) propagation conditions within their own bands. Throughout the paper, the subcarrier index is marked with $c$ and the total amount of subcarriers with $C$, and consequently $c \in\{-C / 2, \ldots,-1,1, \ldots, C / 2\}$. The image (or mirror) carrier is defined as $c^{\prime}=-c$. Additionally, we mark the baseband equivalent signal snapshot at subcarrier $c$ as $s_{c}$ and the signal at the image carrier as $s_{c^{\prime}}$.

When I/Q imbalance occurs in OFDM TX, the transmitted baseband equivalent signal snapshot at subcarrier $c$ equals [16]

$$
s_{\mathrm{Txi}, c}=s_{c} K_{\mathrm{Tx} 1, c}+s_{c^{\prime}}^{*} K_{\mathrm{Tx} 2, c} .
$$

Here, $K_{\mathrm{Tx} 1, c}$ and $K_{\mathrm{Tx} 2, c}$ are the TX I/Q imbalance coefficients, again at subcarrier $c$, which are given by

$$
K_{\mathrm{Tx} 1, c}=\frac{1+g_{\mathrm{Tx}, c} e^{j \phi_{\mathrm{Tx}, c}}}{2} \quad \text { and } \quad K_{\mathrm{Tx} 2, c}=\frac{1-g_{\mathrm{Tx}, c} e^{j \phi_{\mathrm{Tx}, c}}}{2}
$$

where $g_{\mathrm{Tx}, c}$ and $\phi_{\mathrm{Tx}, c}$ are the gain and phase imbalance parameters, respectively [5]. Note that ideally $g_{\mathrm{Tx}, c}=1$ and $\phi_{\mathrm{Tx}, c}=0$ and the transmitted signal snapshot reduces to $s_{c}$. Based on (1), we can note that the transmitted signal at subcarrier $c$ is actually consisting of the desired signal but also of the signal meant to be transmitted at the image carrier.

When the transmitted signal snapshot, affected by TX I/Q imbalance, is received by an antenna array with $N$ antenna elements, the received baseband equivalent signal snapshots $\mathbf{r}_{\mathrm{Txi}, c}=\left[r_{\mathrm{Txi}, 1, c}, r_{\mathrm{Txi}, 2, c}, \ldots, r_{\mathrm{Txi}, N, c}\right] \in \mathbb{C}^{N \times 1}$ can be presented as

$$
\mathbf{r}_{\mathrm{Txi}, c}=s_{\mathrm{Txi}, c} \mathbf{h}_{c}+\mathbf{z}_{c}=s_{c} K_{\mathrm{Tx} 1, c} \mathbf{h}_{c}+s_{c^{\prime}}^{*} K_{\mathrm{Tx} 2, c} \mathbf{h}_{c}+\mathbf{z}_{c}
$$

where a perfect synchronization between TX and RX is assumed. Firstly, $\mathbf{h}_{c} \in \mathbb{C}^{N \times 1}$ consists of channel responses between the TX antenna and each of the RX antennas. We don't assume any specific spatial correlation for the channel responses and thus all formulations are valid in general. In addition, throughout the paper, the channel response elements as well as the I/Q imbalance parameters are assumed to be
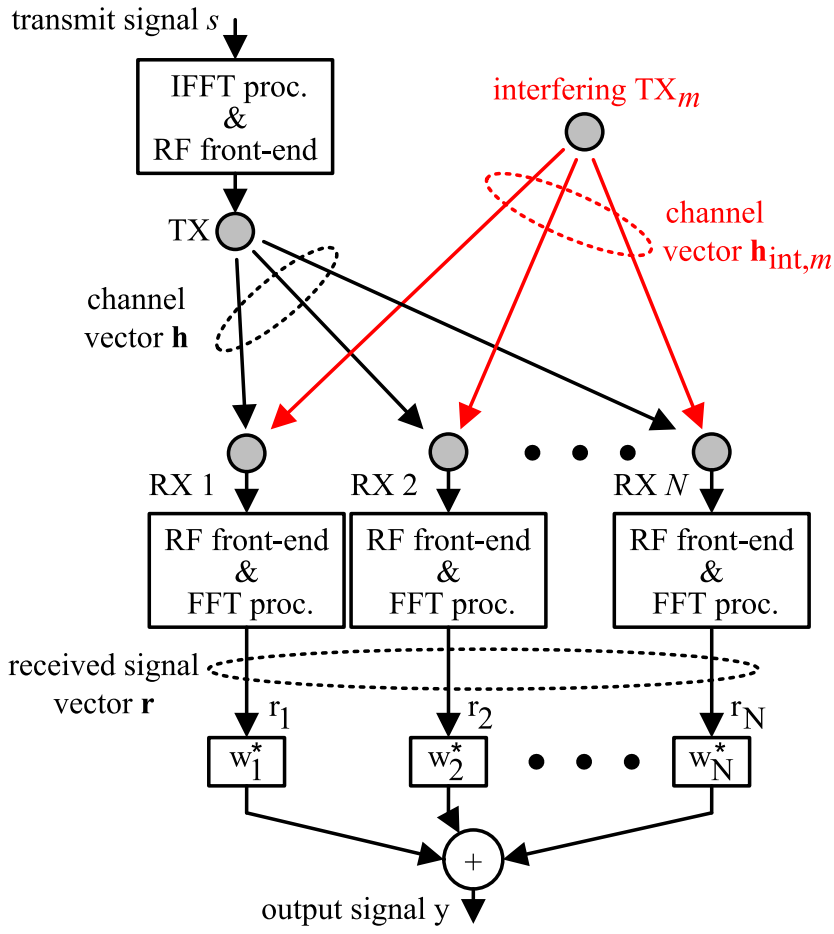

Fig. 1. A simplified illustration of considered scenario. All variables represents signals, responses, or coefficients at an arbitrary OFDM subcarrier.

constant within each of the narrow subcarrier bands. Secondly, $\mathbf{z}_{c} \in \mathbb{C}^{N \times 1}$ is the interference plus noise vector at subcarrier $c$ and equals

$$
\mathbf{z}_{c}=\sum_{m=1}^{M} s_{\mathrm{int}, c, m} \mathbf{h}_{\mathrm{int}, c, m}+\mathbf{n}_{c} .
$$

Here, $s_{\text {int }, c, m}$ and $\mathbf{h}_{\text {int }, c, m} \in \mathbb{C}^{N \times 1}$ represent signal snapshot and the channel response vector of the $m^{\text {th }}$ interfering signal, respectively. The total amount of interfering signals equals $M$ and the noise vector $\mathbf{n}_{c} \in \mathbb{C}^{N \times 1}$ models the additive white Gaussian noise in the RX electronics. Noise elements in different RX branches are assumed to be complex circular and mutually uncorrelated. A complete transmission chain with an interferer is depicted in Fig. 1.

Next we consider that I/Q imbalance occurs also on the $\mathrm{RX}$ side. For convenience, we first define RX I/Q imbalance coefficient matrices $\left(\in \mathbb{C}^{N \times N}\right)$ given by

$$
\begin{aligned}
& \mathbf{K}_{\mathrm{Rx} 1, c}=\operatorname{diag}\left(K_{\mathrm{Rx} 1,1, c}, \cdots, K_{\mathrm{Rx} 1, N, c}\right) \\
& \mathbf{K}_{\mathrm{Rx} 2, c}=\operatorname{diag}\left(K_{\mathrm{Rx} 2,1, c}, \cdots, K_{\mathrm{Rx} 2, N, c}\right)
\end{aligned}
$$

where the RX I/Q imbalance coefficients of the $n^{\text {th }}$ individual $\mathrm{RX}$ branch are equal to [5]

$$
\begin{aligned}
K_{\mathrm{Rx} 1, n, c} & =\frac{1+g_{\mathrm{Rx}, n, c} e^{-j \phi_{\mathrm{Rx}, n, c}}}{2} \\
K_{\mathrm{Rx} 2, n, c} & =\frac{1-g_{\mathrm{Rx}, n, c} e^{j \phi_{\mathrm{Rx}, n, c}}}{2}
\end{aligned}
$$

where $g_{\mathrm{Rx}, n, c}$ and $\phi_{\mathrm{Rx}, n, c}$ are now the gain and phase imbalance parameters of the $n^{\text {th }}$ RX branch at subcarrier $c$. Then, the 
received signal snapshots $\mathbf{r}_{\mathrm{TxRxi}, c} \in \mathbb{C}^{N \times 1}$ under joint $\mathrm{TX}+\mathrm{RX}$ I/Q imbalance are given by

$$
\begin{aligned}
\mathbf{r}_{\mathrm{TxRxi}, c} & =\mathbf{K}_{\mathrm{Rx} 1, c} \mathbf{r}_{\mathrm{Txi}, c}+\mathbf{K}_{\mathrm{Rx} 2, c} \mathbf{r}_{\mathrm{Txi}, c^{\prime}}^{*} \\
& =s_{c} \mathbf{K}_{\mathrm{Rx}, c} \tilde{\mathbf{K}}_{\mathrm{TxA}, c} \tilde{\mathbf{h}}_{c}+s_{c^{\prime}}{ }^{\prime} \mathbf{K}_{\mathrm{Rx}, c} \tilde{\mathbf{K}}_{\mathrm{TxB}, c} \tilde{\mathbf{h}}_{c}+\mathbf{K}_{\mathrm{Rx}, c} \tilde{\mathbf{z}}_{c}
\end{aligned}
$$

where the augmented channel response vector $\tilde{\mathbf{h}}_{c}=\left[\mathbf{h}_{c}^{T}, \mathbf{h}_{c^{\prime}}^{H}\right]^{T} \in \mathbb{C}^{2 N \times 1}$, the augmented interference plus noise vector $\tilde{\mathbf{z}}_{c}=\left[\mathbf{z}_{c}^{T}, \mathbf{z}_{c^{\prime}}^{H}\right]^{T} \in \mathbb{C}^{2 N \times 1}$ and the $\mathrm{RX}$ I/Q imbalance matrix $\mathbf{K}_{\mathrm{Rx}, c}=\left[\mathbf{K}_{\mathrm{Rx} 1, c}, \mathbf{K}_{\mathrm{Rx} 2, c}\right] \in \mathbb{C}^{N \times 2 N}$. In addition, the TX I/Q imbalance matrices $\tilde{\mathbf{K}}_{\mathrm{TxA}, c}$ and $\tilde{\mathbf{K}}_{\mathrm{TxB}, c} \in \mathbb{C}^{2 N \times 2 N}$ are defined as

$$
\begin{aligned}
\tilde{\mathbf{K}}_{\mathrm{TxA}, c} & =\left[\begin{array}{cc}
\mathbf{K}_{\mathrm{Tx} 1, c} & \mathbf{0} \\
\mathbf{0} & \mathbf{K}_{\mathrm{Tx} 2, c^{\prime}}^{*}
\end{array}\right] \\
\tilde{\mathbf{K}}_{\mathrm{TxB}, c} & =\left[\begin{array}{cc}
\mathbf{K}_{\mathrm{Tx} 2, c} & \mathbf{0} \\
\mathbf{0} & \mathbf{K}_{\mathrm{Tx} 1, c^{\prime}}^{*}
\end{array}\right] .
\end{aligned}
$$

Here $\mathbf{K}_{\mathrm{Tx} 1, c}=K_{\mathrm{Tx} 1, c} \mathbf{I}_{N}$ and $\mathbf{K}_{\mathrm{Tx} 2, c}=K_{\mathrm{Tx} 2, c} \mathbf{I}_{N} \in \mathbb{C}^{N \times N}$. This is the general form of the received signal under joint TX+RX I/Q imbalance. Throughout the paper, the special case with I/Q imbalance only in the TX can be obtained from the signal models by substituting $\mathbf{K}_{\mathrm{Rx} 1, c}=\mathbf{I}$ and $\mathbf{K}_{\mathrm{Rx} 2, c}=\mathbf{0}$ for all $c$. Similarly, the case with I/Q imbalance only in the RX is obtained by substituting $K_{\mathrm{Tx} 1, c}=1$ and $K_{\mathrm{Tx} 2, c}=0$ for all $c$.

\section{B. Output Signal of A Linear Digital Combiner}

Receiver array processing is usually implemented with a digital linear combiner. It processes the received signal snapshots with complex weights $\mathbf{w}=\left[w_{1}, w_{2}, \ldots, w_{N}\right]^{T} \in \mathbb{C}^{N \times 1}$ and the resulting output signal $y$ can be presented in a convenient inner product format as [17]

$$
y=\mathbf{w}^{H} \mathbf{r} \text {. }
$$

The combiner weights can be selected/solved with blind or non-blind methods, depending on a priori information, under given optimization criteria.

For the case of joint $\mathrm{TX}+\mathrm{RX}$ I/Q imbalance, combining results in an output signal at subcarrier $c$ that is equal to

$$
\begin{aligned}
y_{\mathrm{TxRxi}, c}= & \mathbf{w}_{c}^{H} \mathbf{r}_{\mathrm{TxRxi}, c} \\
= & s_{c} \mathbf{w}_{c}^{H} \mathbf{K}_{\mathrm{Rx}, c} \tilde{\mathbf{K}}_{\mathrm{TxA}, c} \tilde{\mathbf{h}}_{c}+s_{c^{\prime}}{ }^{\prime} \mathbf{w}_{c}^{H} \mathbf{K}_{\mathrm{Rx}, c} \tilde{\mathbf{K}}_{\mathrm{TxB}, c} \tilde{\mathbf{h}}_{c} \\
& +\mathbf{w}_{c}^{H} \mathbf{K}_{\mathrm{Rx}, c} \tilde{\mathbf{z}}_{c} .
\end{aligned}
$$

Here, the first term represents the contribution of the desired signal at subcarrier $c$. The next term consists of the nondesired signal at image carrier $c^{\prime}$ due to I/Q imbalances and can therefore be considered as a self-interference. The last term includes the effects of interfering signals and noise. Note that through $\tilde{\mathbf{z}}_{c}$, the output signal under joint $\mathrm{TX}+\mathrm{RX} \mathrm{I} / \mathrm{Q}$ imbalance includes also a contribution of the interference and noise at the image carrier.

\section{Joint Subcarrier Processing Through Augmented Combiner}

As we saw in the previous subsection, I/Q imbalance causes signal distortion where the signals at subcarriers $c$ and $c^{\prime}$ are mixed with each others. This gives us a motivation for a joint subcarrier processing of the distorted signal. This is obtained by combining the received signal vector $\mathbf{r}_{\mathbf{c}}$ and its conjugated counterpart $\mathbf{r}_{c^{\prime}}^{*}$ from the image carrier with two sets of weights [9], [13], say $\mathbf{w}_{1, c}$ and $\mathbf{w}_{2, c^{\prime}}$. When defining the augmented weight vector as $\tilde{\mathbf{w}}_{c}=\left[\mathbf{w}_{1, c}^{T}, \mathbf{w}_{2, c^{\prime}}^{T}\right]^{T} \in \mathbb{C}^{2 N \times 1}$ and the augmented signal vector as $\tilde{\mathbf{r}}_{c}=\left[\mathbf{r}_{c}^{T}, \mathbf{r}_{c^{\prime}}^{H}\right]^{T} \in \mathbb{C}^{2 N \times 1}$, the output signal of the augmented digital combiner at subcarrier $c$ can be given simply by

$$
\tilde{y}_{c}=\tilde{\mathbf{w}}_{c}^{H} \tilde{\mathbf{r}}_{c} .
$$

Note that although the output has very similar structure as in (12), there is a fundamental difference since now also the signal at the image carrier is included in the processing.

When we define an augmented signal under joint TX+RX I/Q imbalance as $\tilde{\mathbf{r}}_{\mathrm{TxRxi}, c}=\left[\mathbf{r}_{\mathrm{TxRxi}, c}^{T}, \mathbf{r}_{\mathrm{TxRxi}, c^{\prime}}^{H}\right]^{T}$, we can present the output signal of the augmented combiner under joint TX+RX I/Q imbalance as

$$
\begin{aligned}
\tilde{y}_{\mathrm{TxRxi}, c}= & \tilde{\mathbf{w}}_{c}^{H} \tilde{\mathbf{r}}_{\mathrm{TxRxi}, c} \\
= & s_{c} \tilde{\mathbf{w}}_{c}^{H} \tilde{\mathbf{K}}_{\mathrm{Rx}, c} \tilde{\mathbf{K}}_{\mathrm{TxA}, c} \tilde{\mathbf{h}}_{c}+s_{c^{\prime}}^{*} \tilde{\mathbf{w}}_{c}^{H} \tilde{\mathbf{K}}_{\mathrm{Rx}, c} \tilde{\mathbf{K}}_{\mathrm{TxB}, c} \tilde{\mathbf{h}}_{c} \\
& +\tilde{\mathbf{w}}_{c}^{H} \tilde{\mathbf{K}}_{\mathrm{Rx}, c} \tilde{\mathbf{z}}_{c} .
\end{aligned}
$$

Here the augmented RX I/Q imbalance matrix $\tilde{\mathbf{K}}_{\mathrm{Rx}, c} \in$ $\mathbb{C}^{2 N \times 2 N}$ is given by

$$
\tilde{\mathbf{K}}_{\mathrm{Rx}, c}=\left[\begin{array}{cc}
\mathbf{K}_{\mathrm{Rx} 1 c} & \mathbf{K}_{\mathrm{Rx} 2} \\
\mathbf{K}_{\mathrm{Rx} 2 c^{\prime}}^{*} & \mathbf{K}_{\mathrm{Rx} 1 c^{\prime}}^{*}
\end{array}\right] .
$$

Clearly, the structures of (15) and (13) are very similar. However, (15) uses twice as many weights as (13) in order to process both subcarrier signals simultaneously. Naturally this yields doubled computational complexity but also gives us more degrees of freedom for obtaining the wanted signal and interference suppression properties even under I/Q imbalance.

\section{AnAlytical Evaluation}

\section{A. Covariance Matrices}

In order to evaluate the performance of the combiners, most notably combiner output SINR, we will next derive formulas for the covariance matrices of the received signals. We assume that the signals at subcarriers $c$ and $c^{\prime}$, the interfering signals and the additive noise are all mutually uncorrelated. In addition, we assume that the interfering signals and noise are complex circular. Then the covariance matrix $\mathbf{R}_{c} \in \mathbb{C}^{N \times N}$ of the received signal under perfect I/Q matching is given by

$$
\mathbf{R}_{c}=\mathbb{E}\left[\mathbf{r}_{c} \mathbf{r}_{c}^{H}\right]=\sigma_{\mathrm{s}, c}^{2} \mathbf{h}_{c} \mathbf{h}_{c}^{H}+\mathbf{R}_{\mathrm{z}, c}
$$

where $\sigma_{\mathrm{s}, c}^{2}=\mathbb{E}\left[\left|s_{c}\right|^{2}\right]$ is the power of the desired signal at subcarrier $c$ and the covariance matrix of the interference plus 
noise equals

$$
\mathbf{R}_{\mathrm{z}, c}=\mathbb{E}\left[\mathbf{z}_{c} \mathbf{z}_{c}^{H}\right]=\sum_{m=1}^{M} \sigma_{\mathrm{int}, c, m}^{2} \mathbf{h}_{\mathrm{int}, c, m} \mathbf{h}_{\mathrm{int}, c, m}^{H}+\sigma_{\mathrm{n}, c}^{2} \mathbf{I}_{N} .
$$

Here, $\sigma_{\mathrm{int}, c, m}^{2}$ and $\sigma_{\mathrm{n}, c}^{2}$ are the powers of the $m^{\text {th }}$ interfering signal and noise, respectively. For future use, we also define $\tilde{\mathbf{R}}_{\mathrm{z}, c}=\mathbb{E}\left[\tilde{\mathbf{z}}_{c} \tilde{\mathbf{z}}_{c}^{H}\right] \in \mathbb{C}^{2 N \times 2 N}$ as the covariance matrix for the augmented interference and noise vector. Under the aforementioned assumptions, it yields

$$
\tilde{\mathbf{R}}_{\mathrm{z}, c}=\left[\begin{array}{cc}
\mathbf{R}_{\mathrm{z}, c} & \mathbf{0} \\
\mathbf{0} & \mathbf{R}_{\mathrm{z}, c^{\prime}}^{*}
\end{array}\right] \text {. }
$$

When considering the effects of joint TX+RX I/Q imbalances, the covariance matrix equals

$$
\begin{aligned}
\mathbf{R}_{\mathrm{TxRxi}, c}= & \mathbb{E}\left[\mathbf{r}_{\mathrm{TxRxi}, c} \mathbf{r}_{\mathrm{TxRxi}, c}^{H}\right] \\
= & \sigma_{\mathrm{s}, c}^{2} \mathbf{K}_{\mathrm{Rx}, c} \tilde{\mathbf{K}}_{\mathrm{TxA}, c} \tilde{\mathbf{h}}_{c} \tilde{\mathbf{h}}_{c}^{H} \tilde{\mathbf{K}}_{\mathrm{TxA}, c}^{H} \mathbf{K}_{\mathrm{Rx}, c}^{H} \\
& +\sigma_{\mathrm{s}, c}^{2} \mathbf{K}_{\mathrm{Rx}, c} \tilde{\mathbf{K}}_{\mathrm{TxB}, c} \tilde{\mathbf{h}}_{c} \tilde{\mathbf{h}}_{c}^{H} \tilde{\mathbf{K}}_{\mathrm{TxB}, c}^{H} \mathbf{K}_{\mathrm{Rx}, c}^{H} \\
& +\mathbf{K}_{\mathrm{Rx}, c} \tilde{\mathbf{R}}_{\mathrm{z}, c} \mathbf{K}_{\mathrm{Rx}, c}^{H} .
\end{aligned}
$$

Here, the first term represents the effect of the desired signal term at subcarrier $c$, whereas the second term corresponds to the self-interference from the image carrier. The last row represents the effect of the interference and noise.

The augmented signal has a slightly more complicated covariance matrix than (20). Fortunately, the covariance matrix of the augmented signal model can be in general expressed as

$$
\tilde{\mathbf{R}}_{c}=\left[\begin{array}{ll}
\mathbf{R}_{c} & \mathbf{C}_{c} \\
\mathbf{C}_{c^{\prime}}^{*} & \mathbf{R}_{c^{\prime}}^{*}
\end{array}\right]
$$

where $\tilde{\mathbf{R}}_{c} \in \mathbb{C}^{2 N \times 2 N}$ and the complementary covariance matrix $\mathbf{C}_{c}=\mathbb{E}\left[\mathbf{r}_{c} \mathbf{r}_{c^{\prime}}^{T}\right] \in \mathbb{C}^{N \times N}$. Therefore, in order to find an expression for the covariance matrix of the augmented signal model, we need to derive the complementary covariance matrix under I/Q imbalance. With ideal RF electronics and assuming that the signals at subcarriers $c$ and $c^{\prime}$ are complex circular and uncorrelated, the complementary covariance matrices become zero matrices. However, as shown in the previous section, I/Q imbalance creates dependencies between the signals at different subcarriers and thus results in noncircular signals, even if the signals have been originally circular. Consequently, the complementary covariance matrix of the received signal under joint TX+RX I/Q imbalance equals

$$
\begin{aligned}
\mathbf{C}_{\mathrm{TxRxi}, c}= & \mathbb{E}\left[\mathbf{r}_{\mathrm{TxRxi}, c} \mathbf{r}_{\mathrm{TxRxi}, c^{\prime}}^{T}\right] \\
= & \sigma_{\mathrm{s}, c}^{2} \mathbf{K}_{\mathrm{Rx}, c} \tilde{\mathbf{K}}_{\mathrm{TxA}, c} \tilde{\mathbf{h}}_{c} \tilde{\mathbf{h}}_{c^{\prime}}^{T} \tilde{\mathbf{K}}_{\mathrm{TxB}, c^{\prime}}^{T} \mathbf{K}_{\mathrm{Rx}, c^{\prime}}^{T} \\
& +\sigma_{\mathrm{s}, c^{\prime}}^{2} \mathbf{K}_{\mathrm{Rx}, c} \tilde{\mathbf{K}}_{\mathrm{TxB}, c} \tilde{\mathbf{h}}_{c} \tilde{\mathbf{h}}_{c^{\prime}}^{T} \tilde{\mathbf{K}}_{\mathrm{TxA}, c^{\prime}}^{T} \mathbf{K}_{\mathrm{Rx}, c^{\prime}}^{T} \\
& +\mathbf{K}_{\mathrm{Rx} 1, c} \mathbf{R}_{\mathrm{z}, c} \mathbf{K}_{\mathrm{Rx} 2, c^{\prime}}^{T}+\mathbf{K}_{\mathrm{Rx} 2, c} \mathbf{R}_{\mathrm{z}, c^{\prime}}^{*} \mathbf{K}_{\mathrm{Rx} 1, c^{\prime}}^{T}
\end{aligned}
$$

Now we obtain the covariance matrix of the augmented signal model under joint TX+RX I/Q imbalance simply by substituting (20) and (22) into (21).

\section{B. Output Powers}

The total output power of the combiner under ideal I/Q matching can be given first simply by [17]

$$
\begin{aligned}
\mathbb{E}\left[\left|y_{c}\right|^{2}\right]= & \mathbf{w}_{c}^{H} \mathbf{R}_{c} \mathbf{w}_{c} \\
= & \sigma_{\mathrm{s}, c}^{2}\left|\mathbf{w}_{c}^{H} \mathbf{h}_{c}\right|^{2}+\sum_{m=1}^{M} \sigma_{\mathrm{int}, c, m}^{2}\left|\mathbf{w}_{c}^{H} \mathbf{h}_{\mathrm{int}, c, m}\right|^{2} \\
& +\sigma_{\mathrm{n}, c}^{2} \mathbf{w}_{c}^{H} \mathbf{I}_{N} \mathbf{w}_{c} .
\end{aligned}
$$

The total output power depends clearly on three terms. The first term represents the contribution of the desired signal. In order to obtain good signal characteristics, the weights should be selected in such a manner that $\left|\mathbf{w}_{c}^{H} \mathbf{h}_{c}\right|$ is maximized. The second term consists of the contributions of the external interferers. This term should naturally be minimized and therefore $\left|\mathbf{w}_{c}^{H} \mathbf{h}_{\text {int }, c, m}\right|$ should be as small as possible for all $m$, i.e. the weights should be orthogonal with all of the interferer channel responses. Finally, the noise contributes the total output power simply through the noise power, having neither channel nor spatial dependency involved.

When substituting (20) into (23) we get the output power under joint TX+RX I/Q imbalance as

$$
\begin{aligned}
\mathbb{E}\left[\left|y_{\mathrm{TxRxi}, c}\right|^{2}\right]= & \mathbf{w}_{c}^{H} \mathbf{R}_{\mathrm{TxRxi}, c} \mathbf{w}_{c} \\
= & \sigma_{\mathrm{s}, c}^{2}\left|\mathbf{w}_{c}^{H} \mathbf{K}_{\mathrm{Rx}, c} \tilde{\mathbf{K}}_{\mathrm{TxA}, c} \tilde{\mathbf{h}}_{c}\right|^{2} \\
& +\sigma_{\mathrm{s}, c^{\prime}}^{2}\left|\mathbf{w}_{c}^{H} \mathbf{K}_{\mathrm{Rx}, c} \tilde{\mathbf{K}}_{\mathrm{TxB}, c} \tilde{\mathbf{h}}_{c}\right|^{2} \\
& +\mathbf{w}_{c}^{H} \mathbf{K}_{\mathrm{Rx}, c} \tilde{\mathbf{R}}_{\mathrm{z}, c} \mathbf{K}_{\mathrm{Rx}, c}^{H} \mathbf{w}_{c} .
\end{aligned}
$$

Again, the first term corresponds to the desired signal term whereas the second term represents the influence of the selfinterference. The last term includes the contribution of the external interference and noise.

The power for the output signal of the augmented combiner is similar to (24) but the weights as well as the covariance matrix have to be replaced with their augmented counterparts. The resulting output power of the augmented signal under joint $\mathrm{TX}+\mathrm{RX} \mathrm{I} / \mathrm{Q}$ imbalance is then given simply by

$$
\begin{aligned}
\mathbb{E}\left[\left|\tilde{y}_{\mathrm{TxRx}, c}\right|^{2}\right]= & \tilde{\mathbf{w}}_{c}^{H} \tilde{\mathbf{R}}_{\mathrm{TxRxi}, c} \tilde{\mathbf{w}}_{c} \\
= & \sigma_{\mathrm{s}, c}^{2}\left|\tilde{\mathbf{w}}_{c}^{H} \tilde{\mathbf{K}}_{\mathrm{Rx}, c} \tilde{\mathbf{K}}_{\mathrm{TxA}, c} \tilde{\mathbf{h}}_{c}\right|^{2} \\
& +\sigma_{\mathrm{s}, c^{\prime}}^{2}\left|\tilde{\mathbf{w}}_{c}^{H} \tilde{\mathbf{K}}_{\mathrm{Rx}, c} \tilde{\mathbf{K}}_{\mathrm{TxB}, c} \tilde{\mathbf{h}}_{c}\right|^{2} \\
& +\tilde{\mathbf{w}}_{c}^{H} \tilde{\mathbf{K}}_{\mathrm{Rx}, c} \tilde{\mathbf{R}}_{\mathrm{z}, c} \tilde{\mathbf{K}}_{\mathrm{Rx}, c}^{H} \tilde{\mathbf{w}}_{c} .
\end{aligned}
$$

\section{Signal to Interference and Noise Ratios}

In order to evaluate the performance of the conventional and augmented combiners under I/Q imbalance, we will next formulate SINR expressions for the combiner output signals. SINRs illustrate the performance of the combiners from a practical point of view, while also offering a commonly used metric for comparisons with other studies in the literature. 
SINRs are easily derived from the output signal powers in (24)-(25), since we have grouped them conveniently already in the previous subsection. As a result, SINR expression for the conventional combiner is given by (26), whereas SINR for the augmented combiner is given by (27).

$$
\begin{array}{r}
\operatorname{SINR}_{\mathrm{TxRxi}, c}=\frac{\sigma_{\mathrm{s}, c}^{2}\left|\mathbf{w}_{c}^{H} \mathbf{K}_{\mathrm{Rx}, c} \tilde{\mathbf{K}}_{\mathrm{TxA}, c} \tilde{\mathbf{h}}_{c}\right|^{2}}{\left\{\sigma_{\mathrm{s}, c^{\prime}}^{2}\left|\mathbf{w}_{c}^{H} \mathbf{K}_{\mathrm{Rx}, c} \tilde{\mathbf{K}}_{\mathrm{TxB}, c} \tilde{\mathbf{h}}_{c}\right|^{2}+\right.} \\
\left.\mathbf{w}_{c}^{H} \mathbf{K}_{\mathrm{Rx}, c} \tilde{\mathbf{R}}_{\mathrm{z}, c} \mathbf{K}_{\mathrm{Rx}, c}^{H} \mathbf{w}_{c}\right\} \\
\widetilde{\operatorname{SINR}}_{\mathrm{TxRxi}, c}=\frac{\sigma_{\mathrm{s}, c}^{2}\left|\tilde{\mathbf{w}}_{c}^{H} \tilde{\mathbf{K}}_{\mathrm{Rx}, c} \tilde{\mathbf{K}}_{\mathrm{TxA}, c} \tilde{\mathbf{h}}_{c}\right|^{2}}{\left\{\sigma_{\mathrm{s}, c^{\prime}}^{2}\left|\tilde{\mathbf{w}}_{c}^{H} \tilde{\mathbf{K}}_{\mathrm{Rx}, c} \tilde{\mathbf{K}}_{\mathrm{TxB}, c} \tilde{\mathbf{h}}_{c}\right|^{2}+\right.} \\
\left.\tilde{\mathbf{w}}_{c}^{H} \tilde{\mathbf{K}}_{\mathrm{Rx}, c} \tilde{\mathbf{R}}_{\mathrm{z}, c} \tilde{\mathbf{K}}_{\mathrm{Rx}, c}^{H} \tilde{\mathbf{w}}_{c}\right\}
\end{array}
$$

Notice that, through $\tilde{\mathbf{R}}_{\mathrm{z}, c}$, (26) and (27) are affected by the interference and noise from the desired subcarrier but also from the image carrier. Obviously this non-desired behavior, caused by I/Q imbalance, creates additional challenges for the combiner weight selection. In the next subsection, we will discuss the weight selection problem and formulate two solution methods for the weights. We will also show that due to the doubled degrees of freedom, the augmented combiner suppresses the interference and noise more efficiently than the conventional per-subcarrier processing.

\section{OPtimal MMSE COMBINER}

A well-known statistical method for solving stationary estimation problems is the so-called Wiener filter. It is an optimal solution in the MMSE sense [2] and the corresponding solution for the weight selection problem at hand is given by

$$
\mathbf{w}_{\mathrm{MMSE}, c}=\mathbf{R}_{c}^{-1} \mathbf{p}_{c}
$$

where $\mathbf{p}_{c}=\mathbb{E}\left[s_{c}^{*} \mathbf{r}_{c}\right] \in \mathbb{C}^{N \times 1}$ is the cross-correlation vector between the desired signal and the received signal snapshots. The cross-correlation vector under joint TX+RX I/Q imbalance equals

$$
\mathbf{p}_{\mathrm{TxRxi}, c}=\mathbb{E}\left[s_{c}^{*} \mathbf{r}_{\mathrm{TxRxi}, c}\right]=\sigma_{s, c}^{2} \mathbf{K}_{\mathrm{Rx}, c} \tilde{\mathbf{K}}_{\mathrm{TxA}, c} \tilde{\mathbf{h}}_{c} .
$$

Unfortunately, the Wiener combiner is sensitive to I/Q imbalance leading to performance degradation [4]. To overcome this problem, the augmented Wiener combiner can be used since it is structurally capable of the joint subcarrier processing. The optimal solution is then given by

$$
\tilde{\mathbf{w}}_{\mathrm{MMSE}, c}=\tilde{\mathbf{R}}_{c}^{-1} \tilde{\mathbf{p}}_{c}
$$

which is very similar to (28) but now all variables are given in the augmented form. The augmented cross-correlation vector $\left(\in \mathbb{C}^{2 N \times 2 N}\right)$ under I/Q imbalance equals now

$$
\tilde{\mathbf{p}}_{\mathrm{TxRxi}, c}=\mathbb{E}\left[s_{c}^{*} \tilde{\mathbf{r}}_{\mathrm{TxRxi}, c}\right]=\left[\begin{array}{c}
\sigma_{s, c}^{2} \mathbf{K}_{\mathrm{Rx}, c} \tilde{\mathbf{K}}_{\mathrm{TxA}, c} \tilde{\mathbf{h}}_{c} \\
\sigma_{s, c}^{2} \mathbf{K}_{\mathrm{Rx}, c^{\prime}}^{*} \tilde{\mathbf{K}}_{\mathrm{TxB}, c^{\prime}}^{*} \tilde{\mathbf{h}}_{c^{\prime}}^{*}
\end{array}\right] .
$$

Wiener combiners would result in optimal MMSE solutions, but the exact statistical information, i.e. $\mathbf{R}_{c}$ and $\mathbf{p}_{c}$, is rarely available. Fortunately, Wiener combiner can be approximated with adaptive data-aided methods [18] which adapt to the current channel conditions and RF imperfections with the help of beacons or pilot signals. One of these methods is least mean squares (LMS) [19] which converges close to Wiener solution. In addition, LMS does not require computationally complex matrix inversions and therefore leads to a solution with lower complexity than the exact Wiener solution. In order to avoid input signal power dependency, we selected to illustrate the results with normalized LMS (NLMS) [19] which can adapt to varying input powers in a more flexible way than the conventional LMS. One should note that (N)LMS is only one of the many applicable adaptive methods. E.g. if faster convergence is wanted, one could use the recursive least squares (RLS) algorithm [19] but this would also result in higher complexity and computational power.

The adaptive augmented NLMS algorithm for the augmented signal model is given by

$$
\begin{aligned}
\tilde{y}_{c}(i) & =\tilde{\mathbf{w}}_{\mathrm{NLMS}, c}^{H}(i) \tilde{\mathbf{r}}_{c}(i) \\
\tilde{e}_{c}(i) & =s_{c}(i)-\tilde{y}_{c}(i) \\
\tilde{\mathbf{w}}_{\mathrm{NLMS}, c}(i+1) & =\tilde{\mathbf{w}}_{\mathrm{NLMS}}(i)+\tilde{\mu} \frac{\tilde{\mathbf{r}}_{c}(i) \tilde{e}_{c}^{*}(i)}{\left\|\tilde{\mathbf{r}}_{c}(i)\right\|^{2}}
\end{aligned}
$$

where $\tilde{\mathbf{w}}_{\mathrm{NLMS}, c} \in \mathbb{C}^{2 N \times 1}$ denotes the augmented NLMS weights, $\tilde{e}_{c}$ is the estimation error and $\tilde{\mu}$ stands for the stepsize coefficient. All parameters, excluding $\tilde{\mu}$, present the values on the $i^{\text {th }}$ iteration round. The weights can be initialized e.g. with all-zeros or with a priori information if available. A corresponding algorithm for the conventional signal models is obtained by replacing all variables in (32)-(34), excluding $s_{c}$, with their non-augmented counterparts. In the next section we evaluate the performance of the combiners numerically.

\section{Numerical Evaluations}

In numerical evaluations we model a setup where one TX transmits an OFDM signal waveform which is received by an antenna array consisting of eight antenna elements. Mutually independent 16-quadrature amplitude modulation (QAM) symbols are deployed as subcarrier modulation. We also add four complex circular Gaussian waveforms to the received signals for modeling the contribution of external interference from other, non-desired, users. The transmission channels between all TX-RX antenna pairs as well as between all interferer-RX antenna pairs are independent and Rayleigh distributed. Finally, we add additive white Gaussian noise on top of the received signals for modeling the noise in the RX electronics. The noise power in all RX branches is assumed to be the same. We define the signal to noise ratio (SNR) as a ratio of the averaged received signal power (per RX antenna) and noise power. The signal to interference ratio (SIR) is defined as a ratio of the received signal power and the total received interference power. 


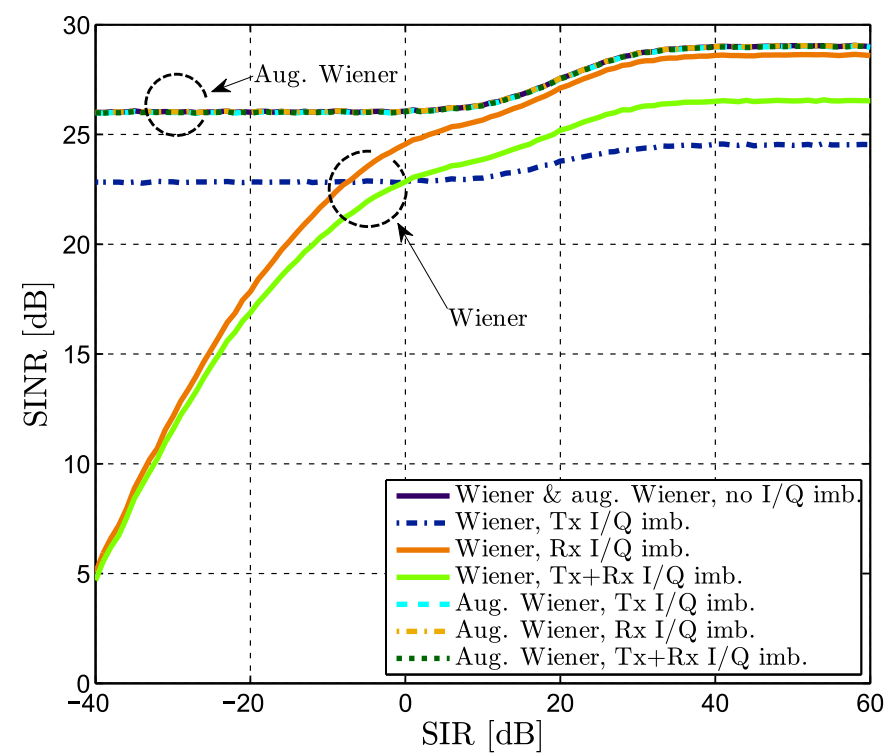

Fig. 2. SINR as a function of SIR when all four interferers have equal powers and SNR is fixed to $20 \mathrm{~dB}$. The results are averaged over 10000 realizations. Note that the combiners have equal performance under perfect I/Q matching.

For each realization, the I/Q imbalance gain coefficients $g_{\mathrm{TX}, c}$ and $g_{\mathrm{Rx}, c, i}, i=1, \cdots, N$, are independently selected from $\mathcal{U}(0.9,1.1)$ whereas the phase imbalance coefficients $\phi_{\mathrm{Tx}, c}$ and $\phi_{\mathrm{Rx}, c, i}, i=1, \cdots, N$, are independently selected from $\mathcal{U}\left(-10^{\circ}, 10^{\circ}\right)$. The I/Q imbalance parameters at different subcarriers are assumed to be independent for modeling arbitrarily frequency selective I/Q imbalance.

Fig. 2 depicts the SINR as a function of the SIR for Wiener combiners under different I/Q imbalance scenarios. We assumed four interferers with equal powers and a fixed $\mathrm{SNR}=20 \mathrm{~dB}$. Evidently, the Wiener combiner suffers from I/Q imbalances and its overall performance is degraded. With low interference levels, i.e. with the high SIRs, the impact of the noise is dominant over the impact of I/Q imbalance and TX I/Q imbalance results in the worst performance. This is well in line with the results in [20]. However, when SIR decreases, the interference becomes more dominant and the performance under either RX I/Q or joint $T X+R X$ I/Q imbalances deteriorate heavily, becoming worse than under TX I/Q imbalance. This is a consequence from the fact that the contribution of the interference and noise depends on RX I/Q imbalance (see (26) and (27)) whereas TX I/Q imbalance affects only the self-interference. This is an essential result and should be taken into consideration when OFDM based systems utilizing antenna arrays are used in the presence of strong interferers. In contrast to the conventional per-subcarrier processing, the augmented Wiener combiner provides good performance in all imbalance cases. The overall performance is flooring at both low and high SIRs, not because of I/Q imbalances but because of the theoretical performance limits of the ideal combiners.

Fig. 3 shows the SINR as a function of SNR for Wiener combiners under different I/Q imbalance scenarios. The total

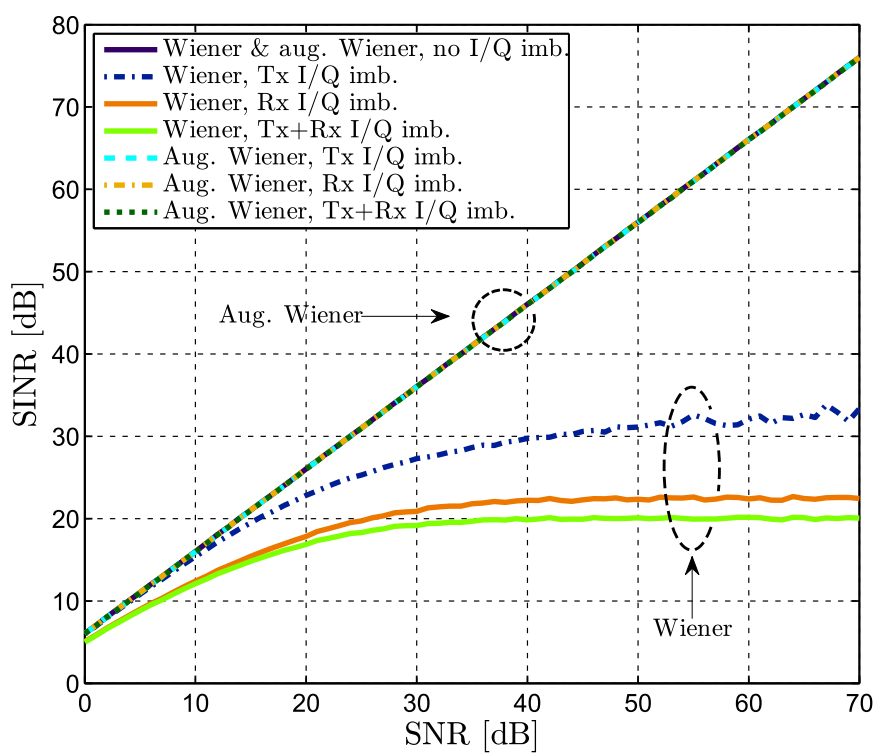

Fig. 3. SINR as a function of SNR when SIR is fixed to $-20 \mathrm{~dB}$. The results are averaged over 10000 realizations. Note that the combiners have equal performance under perfect I/Q matching.

received interference power level is here $20 \mathrm{~dB}$ higher than the desired signal power. Results show that the SINR of the conventional Wiener combiner under I/Q imbalances saturates with low noise levels and hence becomes interference limited. In addition, TX I/Q imbalance is not as harmful as RX I/Q imbalance which extends the results in [12] where a simpler $\mathrm{I} / \mathrm{Q}$ imbalance scenario was used without external interferers. Intuitively, the joint $\mathrm{TX}+\mathrm{RX} \mathrm{I} / \mathrm{Q}$ imbalance leads to the worst performance. Again, in contrast to the conventional Wiener combiner, the augmented Wiener combiner does not suffer from similar performance degradation. Based on the results, it can remove the effects of I/Q imbalance completely and thus results in good overall performance in all imbalance cases.

Finally, we analyze the performance of the practical dataaided NLMS combiners. Fig. 4 shows how the performance of NLMS algorithm is improved as a function of iteration rounds when the SNR is fixed to $20 \mathrm{~dB}$ and the SIR is fixed to $-15 \mathrm{~dB}$. The results show that NLMS indeed converges close to Wiener solutions. In addition, we can note that if RX I/Q imbalance is included in the system, the convergence is slower than in other cases. Fig. 5 depicts the performance of the augmented NLMS algorithm under the same conditions as NLMS in Fig. 4. Clearly, the performance of the augmented NLMS under I/Q imbalances is significantly better than with NLMS. In contrast to the conventional NLMS, the convergence speed is now almost independent of the I/Q imbalance scenario and consequently the convergence under RX and TX+RX I/Q imbalance is much faster than with NLMS. We have further noticed that the total interference plus noise level affects highly the convergence speed of both combiners. E.g. with SIR $=\infty$ and SNR $=20 \mathrm{~dB}$, NLMS converges after 5000 iterations and the augmented NLMS is even faster, converging already after 150 iterations under all I/Q imbalance scenarios. 


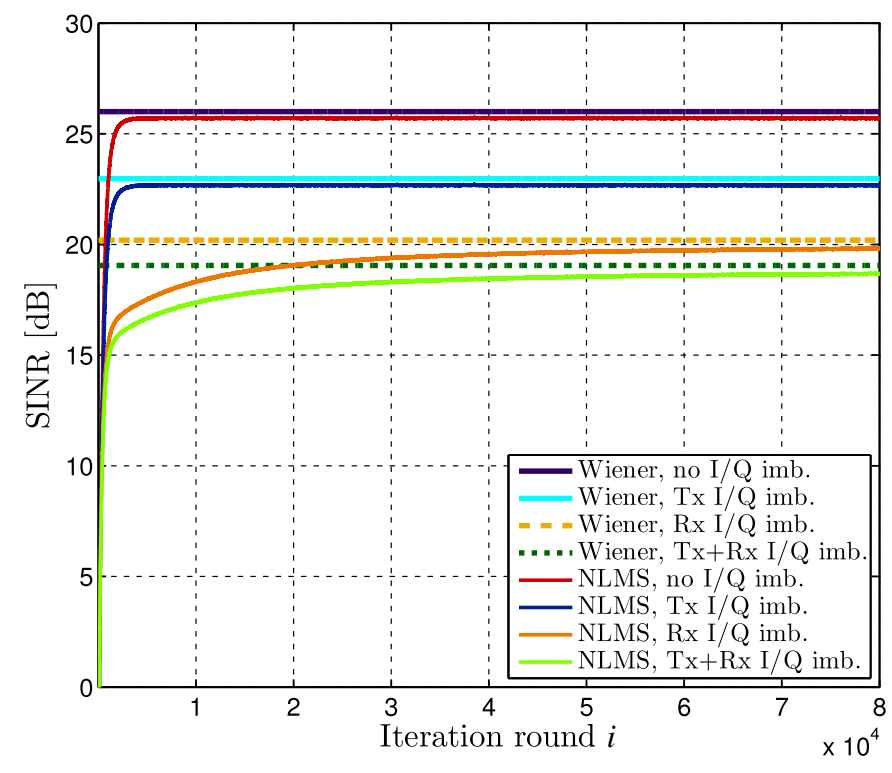

Fig. 4. Convergence of SINR with the conventional combiner. SNR is fixed to $20 \mathrm{~dB}$ and SIR is fixed to $-15 \mathrm{~dB}$. The results are averaged over 500 realizations. Step-size parameter $\mu=0.1$. Note the scale of the $\mathrm{x}$-axis.

\section{CONCLUSION}

In this paper, we analyzed the interference suppression with antenna arrays in OFDM systems under transceiver I/Q imbalances. We derived subcarrier level signal models for antenna array combiners in the presence of interferers and under joint TX+RX I/Q imbalances. The analytical evaluation was carrier out with covariance matrices, signal powers and SINRs. Finally, we illustrated the results numerically. All analysis assumed that the I/Q imbalance parameters and the channel responses can be arbitrarily frequency selective. In addition, we didn't assume any specific spatial correlation for the channel responses for keeping the formulations generic.

Based on the theoretical limitations of the conventional persubcarrier processing, we proposed a joint subcarrier processing where the signal at subcarrier $c$ is combined with the signal at the image carrier $c^{\prime}$ and across all antenna elements. Simulations showed that the conventional per-subcarrier processing results in heavy performance degradation under I/Q imbalances, especially in surroundings with high interference levels. In contrast to the per-subcarrier processing, the proposed joint subcarrier processing mitigates I/Q imbalances successfully, also in the presence of strong interferers. It removes the influence of both TX and RX I/Q imbalances and thus enables the same overall performance as if $\mathrm{I} / \mathrm{Q}$ matchings were perfect.

\section{REFERENCES}

[1] S. Haykin, "Cognitive radio: brain-empowered wireless communications," IEEE J. Sel. Areas Commun., vol. 23, no. 2, pp. 201 - 220, Feb. 2005.

[2] H. L. V. Trees, Detection, Estimation, and Modulation Theory, Optimum Array Processing. John Wiley \& Sons, Apr. 2004.

[3] A. Hakkarainen, J. Werner, K. Dandekar, and M. Valkama, "RFaware widely-linear beamforming and null-steering in cognitive radio transmitters," in Proc. CROWNCOM, 2013, pp. 172-177.

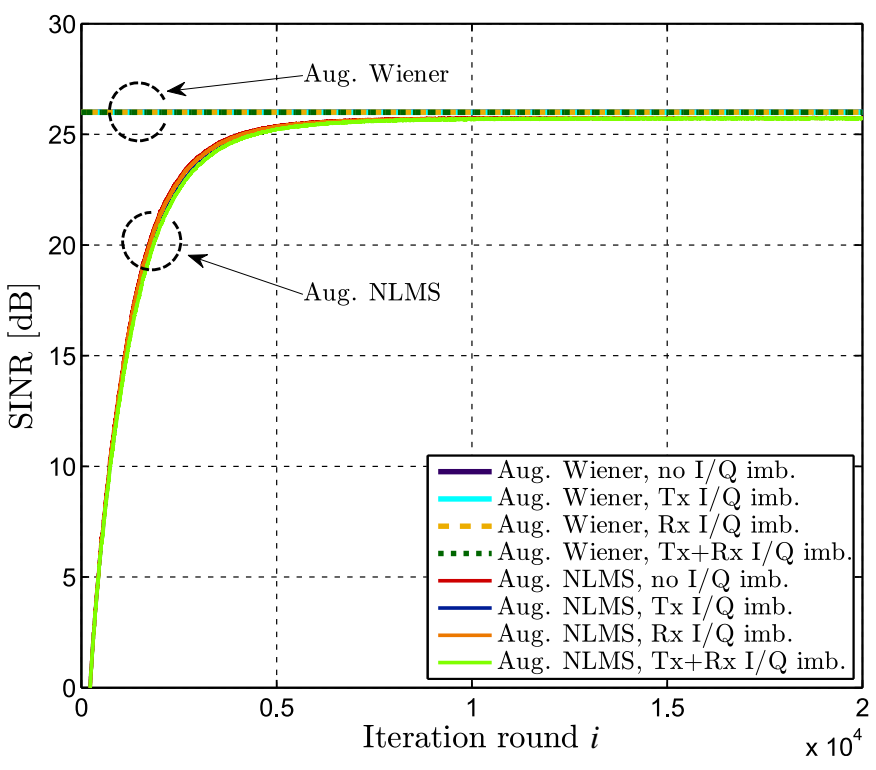

Fig. 5. Convergence of SINR with the augmented combiner. SNR is fixed to $20 \mathrm{~dB}$ and SIR is fixed to $-15 \mathrm{~dB}$. The results are averaged over 500 realizations. Step-size parameter $\tilde{\mu}=0.1$. Note the scale of the $\mathrm{x}$-axis.

[4] A. Hakkarainen, J. Werner, M. Renfors, K. Dandekar, and M. Valkama, "RF-Aware widely-linear MMSE beamforming," in Proc. ISWCS, 2013, pp. 582-586.

[5] T. Schenk, RF Imperfections in High-rate Wireless Systems: Impact and Digital Compensation, 1st ed. Springer, 2008.

[6] J. Cavers and M. Liao, "Adaptive compensation for imbalance and offset losses in direct conversion transceivers," IEEE Trans. Veh. Technol., vol. 42, no. 4, pp. 581-588, 1993.

[7] A. Mashmour, W. Domino, and N. Beamish, "On the direct conversion receiver - a tutorial," Microwave Journal, vol. 44, no. 6, pp. 114-128, Jun. 2001.

[8] H. Rohling, OFDM: Concepts for Future Communication Systems. Springer, Mar. 2011.

[9] A. Tarighat, R. Bagheri, and A. Sayed, "Compensation schemes and performance analysis of IQ imbalances in OFDM receivers," IEEE Trans. Signal Process., vol. 53, no. 8, pp. 3257-3268, 2005.

[10] A. Tarighat and A. Sayed, "Joint compensation of transmitter and receiver impairments in OFDM systems," IEEE Trans. Wireless Commun., vol. 6, no. 1, pp. 240-247, 2007.

[11] B. Maham, O. Tirkkonen, and A. Hjorungnes, "Impact of transceiver I/Q imbalance on transmit diversity of beamforming OFDM systems," IEEE Trans. Commun., vol. 60, no. 3, pp. 643-648, 2012.

[12] T. C. W. Schenk, E. Fledderus, and P. F. M. Smulders, "Performance impact of IQ mismatch in direct-conversion MIMO OFDM transceivers," in Proc. IEEE RWS, 2007, pp. 329-332.

[13] O. Ozdemir, R. Hamila, and N. Al-Dhahir, "I/Q imbalance in multiple beamforming OFDM transceivers: SINR analysis and digital baseband compensation," IEEE Trans. Commun., vol. 61, no. 5, pp. 1914-1925, 2013.

[14] J. Qi and S. Aissa, "Compensation for HPA nonlinearity and I/Q imbalance in MIMO beamforming systems," in Proc. IEEE WiMob, 2010, pp. 78-82.

[15] D. Tandur and M. Moonen, "Compensation of RF impairments in MIMO OFDM systems," in Proc. IEEE ICASSP, 2008, pp. 3097-3100.

[16] _ - "Joint adaptive compensation of transmitter and receiver IQ imbalance under carrier frequency offset in OFDM-Based systems," IEEE Trans. Signal Process., vol. 55, no. 11, pp. 5246-5252, 2007.

[17] J. Litva, Digital Beamforming in Wireless Communications. Artech House Publishers, Aug. 1996.

[18] B. Widrow et al., "Adaptive antenna systems," Proc. IEEE, vol. 55, no. 12, pp. 2143-2159, Dec. 1967.

[19] S. Haykin, Adaptive Filter Theory, 3rd ed. Prentice Hall, 1995.

[20] O. Özdemir, R. Hamila, and N. Al-Dhahir, "Exact SINR analysis of OFDM systems under joint Tx/RX I/Q imbalance," in Proc. IEEE PIMRC, 2013, pp. 646-650. 